\title{
Neuropsychological correlates of an energy psychology intervention on flight phobia: A MEG single-case study
}

Franck Di Rienzo, Elodie Saruco ${ }^{1}$, Dawson Church $^{2}$, Sébastien Daligault ${ }^{3}$, Claude Delpuech ${ }^{3,4}$, Jean-Michel Gurret ${ }^{1}$, Ursula Debarnot \& Aymeric Guillot ${ }^{1 *}$

${ }^{1}$ Institut Français de Psychologie Énergétique Clinique, 1 Place du Griffon, 69001 Lyon, France.
${ }^{2}$ National Institute for Integrative Healthcare, Fulton, CA, USA.
${ }^{3}$ CERMEP imagerie du vivant, Département MEG, F-69677, Bron, France.
${ }^{4}$ INSERM U1028, CNRS UMR5292, Centre des neurosciences de Lyon, F-69000, Lyon, France.

${ }^{*}$ Corresponding author: Aymeric Guillot, Institut Français de Psychologie Énergétique Clinique, 1 Place du Griffon, 69001 Lyon, France. e-mail: ay.guillot@hotmail.com

Running head: Neuroscience of EFT

\section{Significance statement}

In this single-case MEG pilot study, an Emotional Freedom Technique (EFT) intervention produced regulation of brain regions exhibiting treatment effects in response to conventional psychotherapy and medication. The neural correlates of the threat response were attenuated, and heightened activation of brain frontal executive regions mediating limbic responses appraisal to stressful stimuli was recorded. These pilot results are consistent with the literature indicating that EFT is an evidence-based treatment for phobias. They provide for the first time knowledge regarding the neurophysiological mechanisms underlying the treatment effects. This study pioneers the methodology required to conduct randomized controlled trials.

\section{Abstract}

Aim: Over 100 studies and meta-analyses of Emotional Freedom Techniques (EFT) demonstrate it to be an evidence-based treatment for anxiety, depression, and PTSD. EFT combines elements of common treatments such as exposure and cognitive therapy with the novel ingredient of acupressure. This study used magnetoencephalography (MEG) to measure brain activity in a subject with a severe fear of flying.

Materials and Methods: Before and after treatment and at 4 week follow-up, the participant was presented with both visual images and personal memories that evoked her phobia. These were compared with emotionally neutral controls. Psychometric measures included the Subjective Units of Distress (SUD) scale and Flight-Anxiety Situations questionnaire (FAS). Posttest SUD and FAS scores were reduced compared to the pretest, though gains were maintained on follow-up for SUD scores only.

Results: MEG data revealed an event-related beta desynchronization $(15-30 \mathrm{~Hz})$ during all experimental sessions and conditions. A linear regression analysis showed that heightened activation of a fronto-occipital cortical and cortico-cerebellar network predicted SUD scores. The results are consistent with those found in medication and psychotherapy studies of phobias and anxiety. EFT increased the ability to engage brain's frontal executive regions mediating limbic responses appraisal to stressful stimuli. EFT also downregulated the activity of limbic and cerebellar regions implicated in the fear response.

Conclusion: This study pioneers the methodology required to conduct randomized controlled trials with robust experimental designs, and identifies brain areas that are targets of interest for future research.

Keywords: Anxiety disorders. Emotional freedom techniques. Functional brain imaging. 


\section{Introduction}

Over the course of the last three decades, energy psychology has become a fully-fledged domain within the psychological sciences. Feinstein et al. (28) described energy psychology as: "Physical and cognitive procedures designed to bring about therapeutic shifts in targeted emotions, cognitions, and behaviors", p. 199, adapted from (30).

Energy psychology techniques encompass a wide range of holistic therapies and are used to treat a wide range of mental disorders. These techniques are assumed to tap into the interdependent relationships between the mind, body and spirit (63). In clinical practice, energy psychology techniques usually have the client focus on an emotional issue or memory while the intervention is performed. The treatment varies depending on the type of energy psychology technique selected by the practitioner (45). The most commonly-used method is called "Emotional Freedom Techniques" (EFT) and it combines elements of cognitive and exposure therapies with acupressure applied to certain meridian points identified in Oriental Medicine. It is described in a manual which has been used for research and training since the inception of the method (EFT; (12, 18)). It uses fingertip stimulation of acupuncture meridian end points by having the client tap on them while maintaining mental focus on a stress-inducing stimulus.

In Oriental medicine, acupuncture meridians are believed to regulate the energy flows of the body, hence the term "energy" psychology (25, 27). The inclusion of such techniques introduces theoretical assumptions that are intrinsically distinct from that of conventional psychotherapies as well as the Western biomedical model (26, 27). These therapies have drawn vehement opposition from critics and characterized as "possible threats to the science of psychiatry and psychology" (21, 49; 62). Studies show that opposition to new therapies is usually successful, with only 2 out of 10 empirically demonstrated treatments ever reaching patients, while implementation takes an average of 17 years (13).

Despite these obstacles, the use of EFT has grown exponentially over the past two decades. A study critical of energy psychology surveyed therapists through Listservs such as Acceptance and Commitment Therapy, the Society for the Science of Clinical Psychology, and the Association of Behavioral and Cognitive Therapies, and found 42 were employing these techniques (31). Analysis of Internet traffic shows that over 3 million people visit the 5 most popular EFT web sites each month (11). Despite calls from critics that research be halted (5), over 100 studies of EFT now appear in the literature (Re-
search.EFTuniverse.com). Meta-analyses show EFT to produce large treatment effects for anxiety, depression and post-traumatic stress disorder (PTSD) (for meta-analyses of randomized and non-randomized trials, see (16, 51, 69). Treatment is typically accomplished in attenuated time frames, ranging from one session for phobias to ten sessions for PTSD (11). In some studies, fewer than five sessions are required to remediate PTSD (36, 43). The energy psychology field has had its own journal for over 10 years and a dedicated professional organization for 20 .

While most early studies of EFT used psychological assessments, recent research increasingly employs objective biological measures. In a pioneering triple-blind randomized controlled trial, Church, Yount, and Brooks (14) measured both psychological distress and salivary cortisol levels in 83 subjects receiving either EFT, conventional psychotherapy, or rest. After an hourlong session, the decrease in psychological distress scores was more than twice as great in the EFT group. This was accompanied by a significant reduction in cortisol, as well as a statistically significant interaction between biomarkers and psychological distress. The same team then examined gene expression in veterans with PTSD, and found that as psychological symptoms improved, genes associated with immunity and inflammation were regulated (15). Baseline cortisol declined significantly by $34 \%$ after a weeklong EFT workshop, while salivary immunoglobulin A increased by $131 \%$ (3). These hormonal changes were accompanied by declines in resting heart rate and blood pressure. A study of the effect of a one-hour psychotherapy session found that EFT upregulated 72 genes (47). These included genes associated with many beneficial physiological processes, including the suppression of cancer tumors, regulation of insulin resistance, immunity from opportunistic infections, antiviral activity, synaptic connectivity between neurons, enhancement of male fertility, regenerating white matter in the cerebral cortex, metabolic regulation, neural plasticity, reinforcement of cell membranes, and the reduction of oxidative stress.

As yet there has been little research on the effects of EFT treatment on brain function. This type of research is important because reviews show associations between psychological disorders and abnormal patterns of cerebral processing (23, 42, 48, 64).

In this study, we sought to elucidate the mechanisms of action of EFT as evidenced through brain imaging. The target condition was phobias, a prevalent anxiety disorder (10, 71). Phobia is an anxiety-related mental disorder defined as: "Excessive fear and (...) conscious avoidance of 
phobic stimuli", (42) , p. 311. Phobias are particularly appropriate to study because of the very brief treatment time frames involved when using EFT. Studies show that (a) phobias are typically resolved in a single session; that (b) treatment takes less than an hour, and that (c) treatment effects are maintained over the course of time.

The first such study was performed by Wells et al. (81) and investigated the efficacy of EFT for treating phobias of small animals (e.g., spiders, mice, rats and cockroaches). After an intervention lasting 30 minutes, participants from the EFT group exhibited an increased capacity to approach the animal. Salas et al. (67) replicated and extended the Wells et al. (81) study using a crossover design. Baker and Siegel (6) performed a separate replication that was carefully designed to control for influences that might have confounded the Wells et al. (81) study such as expectancy effects and therapeutic allegiance. After controlling for these further variables, they concluded that the observed effects were due to the treatment and not to experimental artifacts.

Phobic disorders are associated with abnormal patterns of cerebral processing. In a meta-analysis, Ipser et al. (42) reported increased activation of subcortical structures including the amygdala, the thalamus, the insula and the cerebellum in phobic patients when facing stimuli such as images of the feared object. Taking a similar approach, Duval et al. (23) underlined an increased pattern of activity in brain structures processing emotional responses in phobic patients (i.e., the amygdala, the striatum, the insula and the dorsal anterior cingulate cortex), and, concomitantly, decreased activation in brain structures involved in regulatory behaviors (i.e., the medial and dorsolateral prefrontal cortex, the hippocampus and the rostral anterior cingulate cortex). Treatment interventions such as medication and psychotherapy were associated with changes in these regions (42; 23).

The fear of flying is a common phobia, and one often treated using EFT. Flying-related anxiety disorders have a high prevalence, affecting between 10 and $40 \%$ of the population in industrialized countries such as Norway and the United States (20; 24). The present study is the first to use magnetoencephalography (MEG) to examine brain activity before and after an EFT intervention. It examined function in all areas of the brain of a participant with a long-term and severe fear of flying. Her brain activity was monitored before and after exposure to emotionally laden phobic triggers, and again on follow-up. These were correlated with psychological tests.

\section{Methods}

\section{Participants}

The sole participant was a 30 year-old woman with no history of psychiatric or neurologic disorders. She had suffered from a severe phobia of flying for as long she could remember. The diagnosis of flight phobia was established using the Subjective Units of Distress (SUD) scale (83). The SUD measure consists of a Likert rating ranging from 0 ("No anxiety/distress, totally relaxed") to 10 ("Highest anxiety/distress that you have ever felt"). A score of $>8$ is considered phobic, and has been used as an inclusion criterion in previous research (e.g., 67). The participant's SUD score was 9 (i.e., "Extremely anxious/distressed"), and she was not able to travel by airplane at the time of the study. Ethical approval was granted by the local ethics committee, and the participant signed a written informed consent in agreements with the statements of the Declaration of Helsinki (2013).

\section{Experimental design}

We implemented a test-retest design with a followup. Each experimental session involved i) a psychometric assessment of flight phobia, and ii) MEG recordings of brain activity while processing endogenous and exogenous stimuli selected for their potential to elicit flight phobia. The pretest and the posttest were administered before and after an EFT intervention of 30 minutes, while the follow-up test was administered 4 weeks after the posttest (Figure 1A). The EFT intervention was delivered by an independent EFT therapist trained and certified in clinical EFT (11). The participant viewed images and spoke verbal statements both designed to elicit fear of flying.

\section{Experimental sessions}

Psychometric assessment of flight phobia. Both the pretest and posttest MEG recordings were preceded by a psychometric assessment of flight phobia. We used the Flight Anxiety Situation (FAS) questionnaire (79). The FAS assesses anxiety in three dimensions, i) "Anticipatory Anxiety" (14 items, e.g., "The takeoff is announced"), ii) "In-flight Anxiety" (11 items, e.g., "You hear some noises during the flight"), and iii) "Generalized Flight Anxiety scale" (7 items, e.g., "Friends tell you about a flight"). For each item of the three subscales, participants provide a subjective rating on a Likert scale ranging from 1 ("No anxiety") to 5 ("Overwhelming anxiety"). The internal consistency of the FAS is good to excellent with Cronbach's alphas ranging from 0.88 to 0.97 . 


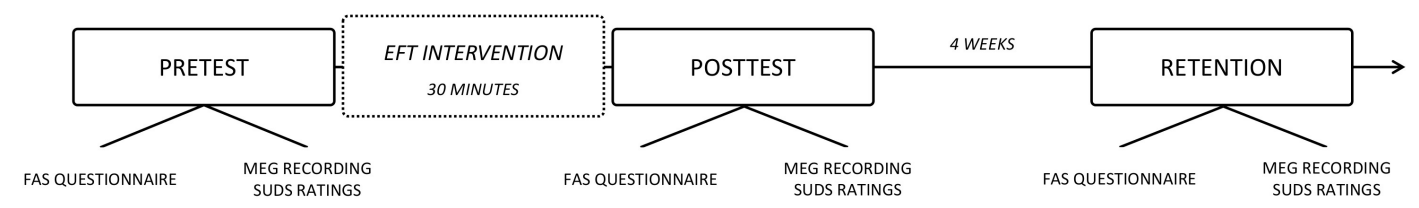

B

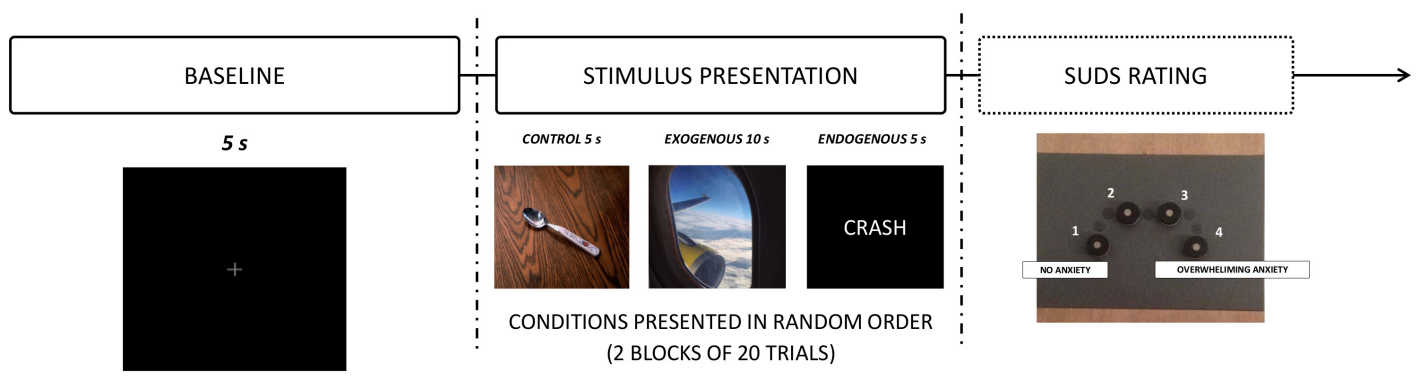

Figure 1: A: Experimental design and experimental measures. B: Timeline of a trial for each experimental condition of the MEG paradigm. FAS: Flight Anxiety Situations questionnaire, MEG: Magnetoencephalography, SUDS: Subjective Units of Distress Scale, EFT: Emotional Freedom Techniques.

The test-retest reliability of the FAS is also excellent, with Pearson's product-moment correlation coefficients ranging from 0.90 to $0.92(79)$.

Magnetoencephalography design. During each experimental session, MEG recordings were taken immediately after the psychometric assessment of flight phobia.

Selection of flight-related pictures. Images and events designed to elicit flight phobia were preselected one week before the first MEG recording session. On a monitor, the participant viewed thirtytwo images of flight-related situations. They included i) planes landing at an airport, ii) the inside and outside of a plane during various phases of flight such as takeoff and landing, and iii) flight incidents and accidents such as fires, collisions and crashes. For each picture, the participant provided a SUD rating. The four pictures yielding the highest SUD ratings were selected as visual stimuli for the exogenous component of the MEG examination.

Selection of flight-related events. We used a 20 minute structured interview to identify participant memories related to flight phobia. Four specific situations in which the participant experienced the highest level of emotional triggering were identified. These were i) the sense of a loss of control during the takeoff phase of flight, ii) the occur- rence of unexpected events during a flight such as turbulence and the vibration of an aircraft's wings, iii) the ground dropping away during takeoff, and iv) an expectation that the plane would crash after takeoff. These self-identified situations were selected for the endogenous component of the MEG examination.

Magnetoencephalography recordings. MEG recordings were performed using a CTF-MEG system (CERMEP, France), with 275 axial gradiometers over the scalp and 29 reference channels for ambient field correction. MEG signals were low-pass filtered $(0-150 \mathrm{~Hz})$ and digitalized at a sampling rate of $600 \mathrm{~Hz}$. Head position was continuously recorded, using three head coils placed on the nasion and pre-auricular points prior to scanning. Brain MRI for co-registration with MEG data was performed using a 1.5T Siemens Magnetom scanner (CERMEP, France - Siemens AG, Erlangen, Germany), and computed using 3-dimentional T1-weighted images encompassing the volume of the entire brain with 1-mm3 cubic voxels (TR: $9.7 \mathrm{~ms}$, TE: $4 \mathrm{~ms}$ ). Extraction and projection of the anatomy of discrete cortical regions using the Montreal Neurologic Institute (MNI) template were performed using the Freesurfer (http://freesurfer.net/) and Brainstorm (74) software packages (http: //neuroimage.usc.edu/brainstorm). 
Magnetoencephalography conditions. The MEG examination consisted of three experimental conditions. During the first experimental condition, the flight-related pictures that produced the highest levels of fear on the SUD scale were displayed on a monitor for 5 seconds. This experimental condition was expected to trigger a phobic response based on an external visual stimulus (the exogenous condition). During the second experimental condition, words related to the participant's phobic memories such as "takeoff" and "crash" were displayed on the monitor. The participant was asked to mentally recall the emotional context associated with the word for 10 seconds. Such recall has been observed to evoke the emotions experienced during an anxiety-producing situation (41), and was expected to generate a phobic response (the endogenous condition). During the third experimental condition, we displayed emotionally neutral pictures extracted from the International Affective Picture System (IAPS) database for 5 seconds each (44). This was expected to control for the emotional response and presence of afferent visual information (the control condition). A baseline of 5 seconds preceded each trial, during which a white cross mark was displayed on the monitor screen. For each experimental condition, the participant provided a SUD rating by pressing a button on a control pad during a 3 second time window (Figure 11B). The control pad used a 4 point Likert scale ranging from 1: "No anxiety" to 4: "Overwhelming anxiety". The MEG captured data from 2 blocks of 20 consecutive trials for each experimental condition, presented in a randomized order, for a total of 40 trials for each experimental condition (Figure 1B).

\section{Dependent variables}

Flight Anxiety Situations scores. We collected FAS scores for "Anticipatory anxiety" (possible score range $=14-70$ ), "In-flight anxiety" (items 1155) and "Generalized flight anxiety" (items 7-35) during the pretest and posttest.

Magnetoencephalography data. Sensor-level analysis. Time-frequency power distributions (0$60 \mathrm{~Hz}$, Morlet wavelets) were normalized with reference to a baseline of 5 seconds (Z-score). The normalized time-frequency maps were then averaged across sensors and trials for each experimental condition. Using a threshold of $|\mathrm{Z}|>2(\mathrm{p}<$ $0.05)$, we estimated the time-frequency windows corresponding to the event-related characteristics of brain rhythms elicited under each experimental condition. Event related desynchronization (ERD) is typically associated with neural activation whereas event-related synchronization (ERS) is associated with neural inhibition and/or resting states (52, 58, 59).

Source analysis. Source reconstruction was obtained by applying a minimum norm inverse solution to the MEG gradiometer signals (4). The dipole orientation was optimized for the reconstruction of cortical and subcortical activity using the "Deep Brain Analysis" feature of the Brainstorm software (1), 2, 74). The minimum norm inverse solution yielded estimates of the time course of cerebral activations at each of the 15000 nodes of the participant's tessellation. A control window ( 5 seconds) provided a baseline prior to the stimulus provided by the exogenous, endogenous and control conditions. The active time window for each measurement was established after the onset of stimulus. The duration of each active time window corresponded to the duration of the ERD or ERS as revealed by sensor-level analysis. We calculated the Power Spectrum Density (PSD) for each source in the active and control time windows, using Welch's method with a $50 \%$ overlap (window length $=1$ second). The PSD frequency range was adjusted to fit the frequency range of the ERD or ERS provided by the sensorlevel analysis. As index of ERD/ERS amplitudes, we calculated a normalized difference between the active and control PSD:

$\mathrm{PSD}_{\mathrm{Norm}}=\frac{\left(P S D_{(\text {Control time window })}-P S D_{(\text {Active time window })}\right)}{\left(P S D_{(\text {Controltimewindow })}+P S D_{(\text {Activetimewindow })}\right)}$

We set the threshold for $\mathrm{PSD}_{\text {Norm }}$ at $5 \%$, and analyzed sequences exhibiting a value $>5 \%$.

Regression analysis. For each experimental condition, we used a random-coefficient regression model that controlled for pretest or posttest SUD ratings by $\mathrm{PSD}_{\text {Norm }}$ calculated during the sourcelevel analysis (1-level linear mixed effects analysis). This analysis was performed at each of the 15000 nodes of the participants' tessellation. This revealed brain sites at which the task-related ERD or ERS amplitudes correlated with SUD ratings in the three experimental conditions.

\section{Statistical analysis}

We used R (176) and (8) to run a linear mixed effects analysis of the FAS scores and SUD ratings recorded throughout the repeated measures design. For FAS scores, we used TEST (Pretest, Posttest, and Follow-UP) and DIMENSION (GENERAL ANXIETy, ANTICIPATORY ANXIETY, and IN-FLIGHT ANXIETY) as fixed effects. As random effect, we used a by-item random intercept. For the MEG SUD ratings, we used TEST (PRETEST, POSTTEST and FOLLOW-UP) and CONDITION (ENDOgEnOUS, ExOgEnOUS and 
CONTROL) as fixed effects with a by-item random intercept corresponding to the picture/event of each trial. Visual inspection of residual plots did not reveal any obvious deviations from homoscedasticity or normality. We reported effect sizes in terms of proportion of variance explained by the linear mixed effects model according to the $\Omega_{0}^{2}$ method (84). The statistical significance threshold was set for a type 1 error rate of $5 \%$. We used contrast tests (least square means difference) for post-hoc investigations and applied the Benjamini-Hochberg step-up procedure to control the false discovery rate (9).

\section{Results}

\section{FAS scores}

The linear mixed effects analysis performed on FAS scores revealed a TEST * DIMENSION interaction $\left(\mathrm{F}(4,64)=3.63, \mathrm{p}<0.01, \Omega_{0}^{2}=0.84\right)$. The ANTICIPATORY ANXIETY scores at PRETEST $(4.00 \pm 0.00)$ were higher than those at POstTest $(2.86 \pm 0.53)(\mathrm{p}<0.001)$. However, there was no difference between PRETEST and FOLLOW-UP $(3.93 \pm 0.27)$ scores $(\mathrm{p}=0.56)$, while POSTTEST scores were lower than FOLLOWUP $(p<0.001)$. Likewise, IN-FLIGHT ANXIETY scores dropped from PRETEST $(3.72 \pm 0.47)$ to Posttest $(3.00 \pm 0.77)(\mathrm{p}<0.001)$, but there was no difference between PRETEST and FOLLOW-UP $(3.72 \pm 0.47)$ scores $(\mathrm{p}=0.99)$ (Figure 2A). FoLLOW-UP scores were higher than POSTTEST $(\mathrm{p}<0.001)$.

The General AnXiety scores at PRETEST (2.86 \pm 0.69$)$ were higher than POstétest $(2.43 \pm 0.43)(\mathrm{p}<0.05)$. However, F OLL OW-UP (3.29 \pm 0.49$)$ scores were higher than both PRETEST and POSTTEST $(\mathrm{p}<$ 0.01 and $\mathrm{p}<0.001$, respectively).

\section{MEG data}

SUD ratings. The linear mixed effects analysis performed on SUD ratings after each trial revealed a TEST $*$ CONDITION interaction $(\mathrm{F}(4,64)=$ $\left.17.61, \mathrm{p}<0.001, \Omega_{0}^{2}=0.63\right)$. For the $\mathrm{EN}_{\mathrm{N}}$ DOGENOUS condition, PRETEST SUD ratings $(2.88 \pm 0.69)$ were higher than both P OsT TEST $(2.10 \pm 0.84)$ and FoLLOW-UP $(1.80 \pm 0.52)$ (both $\mathrm{p}<0.001)$. SUD ratings at FoLLOWUP were lower than P OSTTEST $(\mathrm{p}<0.05)$. For the Exogenous condition, Pretest SUD ratings $(2.85 \pm 0.77)$ were higher than both Posttest $(1.88 \pm 0.61)$ and FolLOW-UP $(1.55 \pm 0.50)$ (both $\mathrm{p}<0.001)$. SUD ratings at FOLLOW-UP were also lower than POSTTEST $(\mathrm{p}<0.01)$ (Figure 2B). No difference was observed between the PRETEST (1.00 \pm 0.00$)$, PostTest $(1.00 \pm 0.00)$ and Follow-UP $(1.00 \pm 0.00)$ SUD ratings for the CONTROL condition (all p >0.05).

Sensor-level analysis. For each experimental condition, we recorded ERD in the beta frequency range $(15-30 \mathrm{~Hz})$ during PRETEST, Post Test and FOLLOW-UP. High amplitudes of highfrequency beta are associated with anxiety and fear. The ERD duration encompassed the $5 \mathrm{~s}$ during which emotionally triggering stimuli were presented to the participant during the $\mathrm{CON}$ TROL and ExOGENOUS conditions. The ERD duration encompassed the $10 \mathrm{~s}$ of stimuli presentation for the ENDOGENOUS condition. The duration of active time windows for the MEG source-level analysis were set up accordingly (see Methods).

Source-level analysis. The primary sources for high-amplitude beta ERD $(15-30 \mathrm{~Hz})$ were in the cortex of the participant's brain. No sources were found in subcortical structures with the exception of the posterior hippocampus during the posttest for ENDOGENOUS stimulation (Figure 3).

During the CONTROL condition, a consistent pattern of primary sensorimotor and right occipital cortex involvement, respectively encompassing Brodmann areas (BA) 4, 6 and 18-19, was recorded for each experimental session (i.e., Pretest, Posttest and Follow-UP) (Figure 3). For the Endogenous condition, the sources of beta ERD were bilaterally distributed in the premotor and primary sensorimotor cortex (BA 4,6) during both PRETEST and the Postente. Notably, cortical involvement was restricted to the left sensorimotor cortex (BA 4, 6) during follow-up. The distribution of beta ERD in the premotor and primary sensorimotor cortex (BA 4,6) was comparable for the exogenous condition across experimental sessions (Figure 3). Additionally, beta ERD was also present in the right posterior hippocampus during the Pretest and the Posttest, but not during the follow-up.

Relationship between beta PSD $_{\text {Norm (15-30 }}$ $\mathbf{H z}$ ) and SUD. During the CONTROL condition, no $\mathrm{PSD}_{\text {Norm }}$ predicted the SUD ratings. Conversely, during ENDOGENOUS, PSD Norm $_{\text {from }}$ the bilateral frontal and occipital cortex, extending to the parietal cortex (BA 11, 39 and 18-19, respectively) predicted SUD ratings (Figure 4). Also, PSD $_{\text {Norm }}$ from the right premotor cortex was associated with the observed variances in SUD ratings. In subcortical structures, SUD ratings were 
A

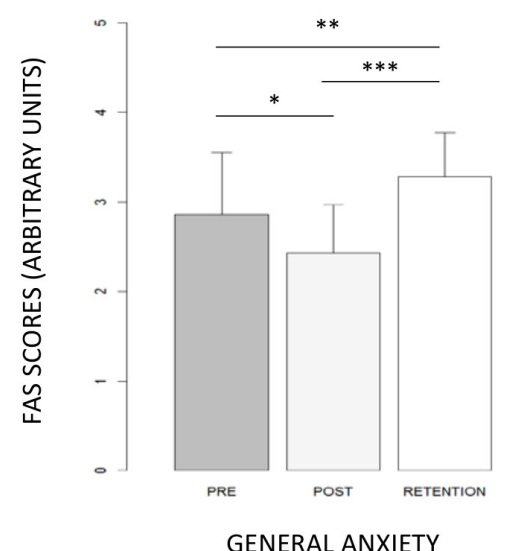

B

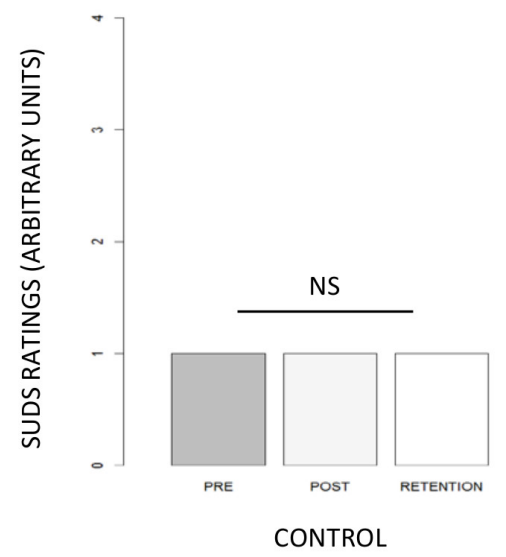

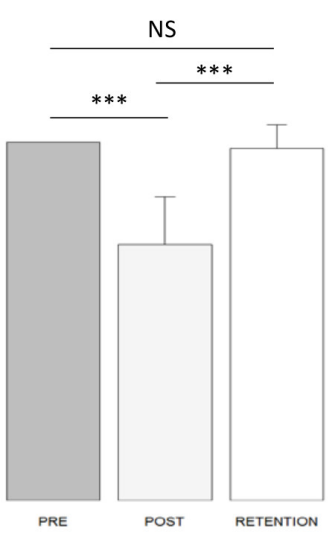

ANTICIPATORY ANXIETY

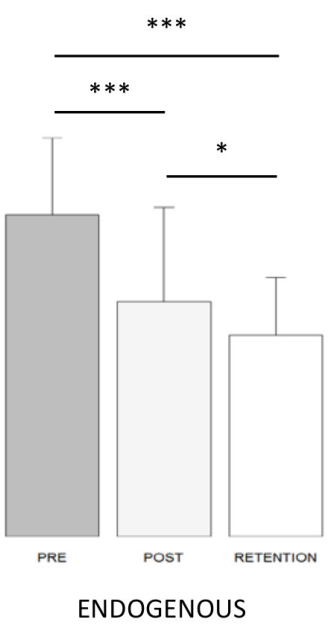

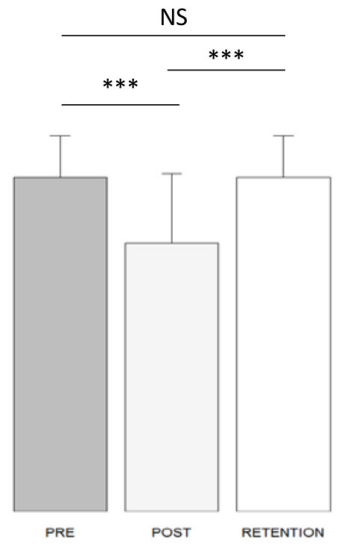

IN-FLIGHT ANXIETY

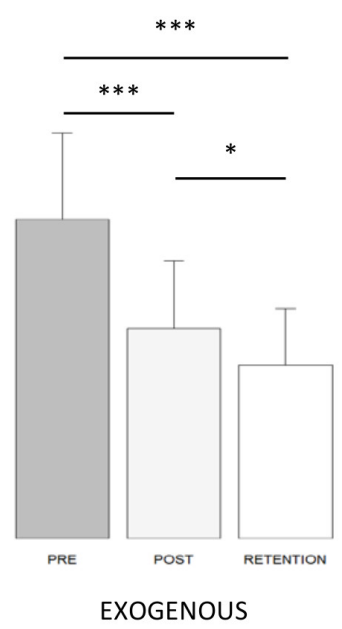

Figure 2: A: Barplot of the TEST by DIMENSION interaction effect on FAS scores. B: Barplot of the TEST by CONDITION interaction effect on SUD ratings provided after each trial of the MEG paradigm. ${ }^{* * *}: \mathrm{p}<0.001,{ }^{* *}: \mathrm{p}<0.01, \mathrm{NS}$ : Not statistically significant difference.

predicted by the $\mathrm{PSD}_{\mathrm{Norm}}$ only from the right cerebellum. During the exogenous condition, a limited number of $\mathrm{PSD}_{\text {Norm }}$ values predicted SUD ratings in cortical structures. This was the case in the left parietal (BA 7) and right premotor (BA 6 ) cortices, and bilaterally in the temporal (BA 38) cortex. In subcortical structures, $\mathrm{PSD}_{\text {Norm }}$ from the bilateral cerebellum, left hippocampus and left amygdala sources predicted SUD (Figure 4).

\section{Discussion}

The present pilot study was designed to examine the neuropsychological correlates of an EFT intervention on flight phobia. Using a single-case design, we used MEG neuroimaging to observe brain function before and after exposure to phobic triggers, with a four-week follow-up. We recorded brain activity when the participant was exposed to triggering memories (the endogenous condition), as well as to disturbing images viewed on a computer monitor (the exogenous condition). Overall, we observed beneficial effects in psychometric measures of flight phobia after the EFT intervention, as well as changes in the activity of the fronto-occipital cortical network and corticocerebellar network that are associated with the extinction of fear. Subjective distress scores (SUD) generally went down after EFT treatment, and dropped further on follow-up. Self-report on the FAS also indicated improvement but follow-up revealed that the gains were not durable.

Like most other studies of EFT for phobias, this study used a brief 30 minute intervention. Posttest FAS scores were substantially reduced 

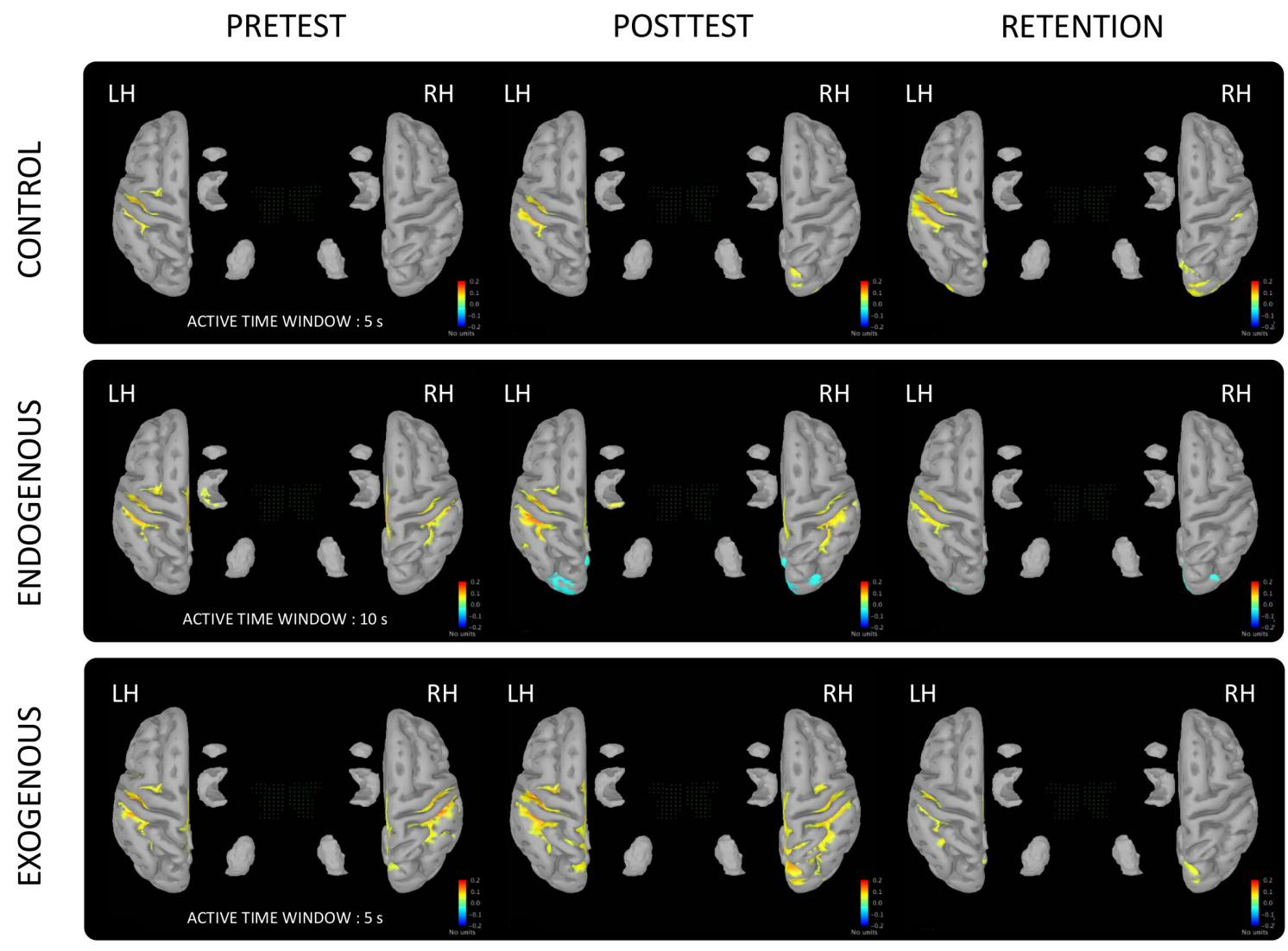

Figure 3: Beta $\mathrm{PSD}_{\text {Norm }}(15-30 \mathrm{~Hz})$ in the source-level, for each experimental session and each experimental condition. Only $\mathrm{PSD}_{\mathrm{Norm}}$ attesting $>5 \%$ of relative difference between active and control time windows are displayed on the map. LH: Left hemisphere, RH: Right hemisphere.

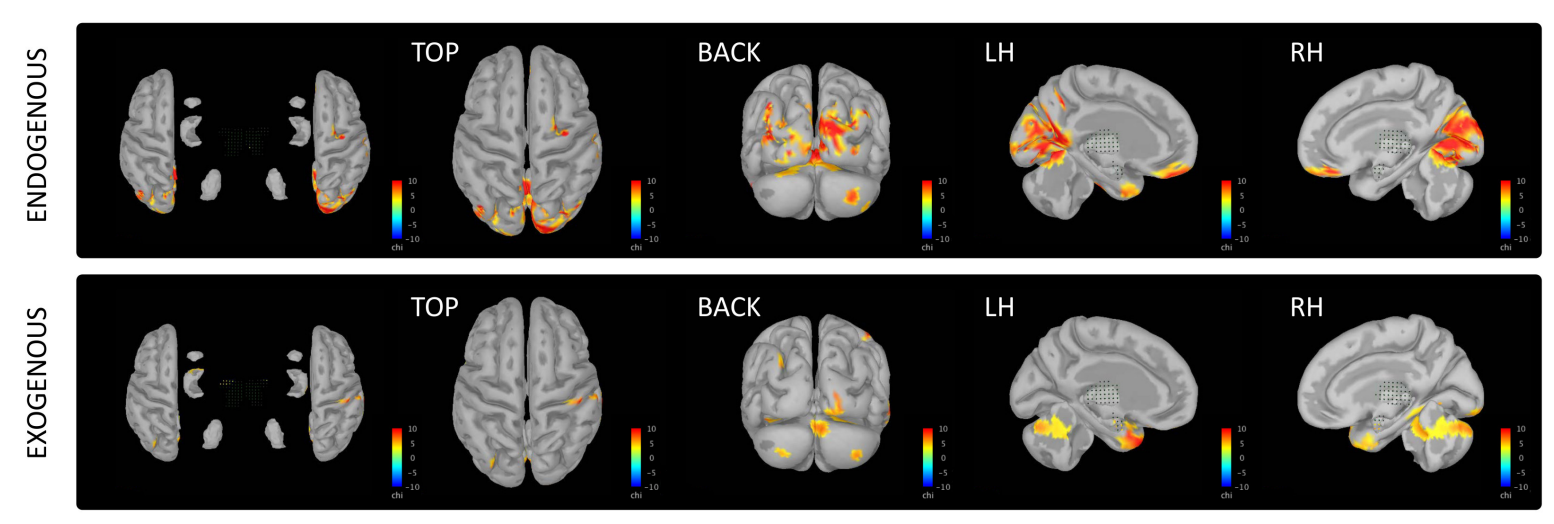

Figure 4: Chi-squared density maps resulting from the regresssion analysis controlling for the TEST effect of SUD ratings by the beta PSD Norm $(15-30 \mathrm{~Hz})$, for each experimental condition. Chisquared values are displayed for a type 1 error rate of $\mathrm{p}<0.05$. The false discovery rate was controlled using the Benjamini-Hochberg step-up procedure implemented in Brainstorm. LH: Left hemisphere, RH: Right hemisphere.

for all dimensions, i.e., general anxiety, anticipatory anxiety, and in-flight anxiety. Likewise, posttest SUD ratings provided after each trial of the endogenous and exogenous conditions were 
significantly reduced. General FAS and SUD ratings attested to immediate beneficial effects corresponding to a decrease in fear of approximately $25 \%$. FAS scores for both anticipatory anxiety and in-flight anxiety returned to pretest values at follow-up while general anxiety scores were higher.

The inverse effect was observed for SUD ratings, which continued to decrease from posttest to follow-up, at which point they dropped to approximately $50 \%$ of their pretest values. While FAS and SUD data provided evidence of positive treatment effects right after the EFT intervention, FAS gains did not persist over time.

We speculate that there are several reasons for this paradox. Flight phobia is a multi-dimensional condition that contains a number of different components $(78,82)$. The FAS questionnaire assesses flight phobia using generic items. It is possible that aspects of the fear of flying other than those targeted by the EFT treatment emerged in the participant's psyche during the four weeks that elapsed before follow-up. This suggests that the phobic components treated during a single brief session may not transfer to other untreated aspects of flight phobia. The process of selecting images and words, in which only images and words identified as triggering by the participant were selected, may have been too specific to generalize the stress-reduction effects to other aspects of flight phobia assessed by the FAS. Also, the treatment may have been too brief. While other studies found that a single session was effective, the severity and long duration of the participant's issue might require a series of treatment sessions rather than a single treatment. Other methods such as cognitive behavioral therapy recommend 12 treatment sessions for phobias (38). The participant might have suffered from anxiety in other life areas as well, and without those being treated they might have raised her general anxiety level in the period between posttest and follow-up. Further research can investigate these possibilities.

The source-level analysis of brain activity revealed that the presentation of fear-producing stimuli consistently engaged the brain motor networks during each experimental session and condition. ERD generators reflect the brain activation related to the cognitive processes induced by the stimulus, and not phase-locked activities evoked by the stimulus presentation (19). During the control condition, the four images selected from the IAPS database were those of innocuous objects such as a wooden box, a spoon, a chair, and a large basin. Presentation of pictures depicting objects represents an input to the brain motor networks $(34,77)$. Since the participant remained motionless during the MEG recording, planning physical interactions with the objects displayed on the screen might have accounted for the activation patterns recorded in BA 4 and 6 (34).

Contrasts in brain activation would therefore not only reflect the emotional processing but also attentional processes related to stimulus perception and encoding. In other words, differences yielded by contrast analyses in functional brain imaging may not systematically relate to the treatment effects recorded at the psychological level. To overcome this limitation, we implemented a linear regression approach to investigate the relationship between brain activity and the psychometric measures of psychological distress. This enabled a statistical mapping of the brain networks predicting the psychological distress measures. This emphasized the brain sources exhibiting a task-related activity corollary of the psychometric recordings along the course of the repeated measures of the design. At the same time, the regression analysis removed task-related brain activity that was not correlated with psychological distress. For the endogenous condition, the regression analysis revealed a bilateral fronto-occipital network extending to parietal brain regions. For the exogenous condition, the regression analysis revealed a bilateral temporo-cerebellar network including the amygdala.

Interestingly, the regression analysis identified changes in brain regions associated with treatment efficacy in phobic disorders in other studies. For the endogenous condition, the regression network did not involve subcortical centers of autobiographic memory, but rather fronto-occipital cortical regions. A review of the literature found that changes in frontal cortex activation are a neural correlate of treatment efficacy in anxiety disorders (for a review, see 23). In social anxiety and PTSD, increased frontal activation is associated with successful treatment (32, 55, 57, 56, 60, 75). Basically, frontal regions play a major role in both the regulation of negative emotions and the process of cognitive appraisal (32, 80). Frontal regions operate through neural pathways involving the subcortical centers that process the threat response, specifically the amygdala (7, 50).

In this study, since the endogenous condition required the recall of mental images, we did not expect to find the occipital cortex serving as a regulator of psychological distress. Yet research into social anxiety disorders shows that the activation of the occipital region correlates with treatment efficacy (22). This might suggest that, during the top-down processing of phobic memories, the participant recruited to a greater extent brain structures responsible for regulatory behaviors, thus yielding greater anxiety appraisal.

For the exogenous condition, we found increased regulation of the amygdala and the cerebellum, 
a pattern noted by others after successful treatment and the reduction of phobic symptoms (42). When confronted with fear-producing images, activation of the amygdala is higher in phobic patients than in controls (39; 40, 68). This is congruent with the role of the amygdala in processing the threat response (53). Ipser et al. (42) emphasized that reduced cerebellum activation was a neural correlate of the beneficial effect of cognitive behavioral therapy on phobias. They noted cerebellar participation in emotional processes, specifically learned and memorized responses to fearful stimuli $(65,66,72$, 73). These findings are in line with epidemiological research on phobias, which finds atypical functioning patterns in cerebello-vestibular structures (46).

The regression analysis performed in the current study also found bilateral involvement of the temporal cortex. This associative region is part of the ventral stream and processes object recognition from visual stimuli (70). Interestingly, dysfunction and atrophy of the temporal lobe has been associated with phobic disorders for quite some time (29). During bottom-up processing of visual stimuli selected for their triggering emotional content, the participant exhibited increased regulation of these temporal lobe regions after EFT treatment.

Finally, the regression analysis revealed the involvement of motor system regions during both the endogenous and exogenous conditions, specifically the premotor and primary sensorimotor cortices. The source-level analysis showed the same pattern. There is a well-established functional coupling between emotional and motor brain systems (61; 35). Emotional priming of the brain's motor systems facilitates readiness to take action on a potential existential threats (17, 37, 54). These fight or flight responses have adaptive survival value (33). More specifically, upregulation of the brain motor system primes the fight or flight response.

The neural correlates of the EFT intervention found in this study overlapped widely with those reported after medication and psychotherapy for anxiety disorders. For the endogenous condition, the participant achieved a more effective cognitive appraisal of the anxiety generated by fearful memories. For the exogenous condition, EFT yielded a reduced emotional threat response when the participant was confronted with visual stimuli selected for their fear-producing effect. Present results are consistent with research showing that EFT regulates the endocrine, cardiovascular and immune systems while producing beneficial epigenetic effects.

As with all research, the present study had a number of limitations. First, the single-case design hampers generalization of the findings due to the absence of a control therapy as well as nonphobic comparison subjects. Placebo, therapist allegiance and demand characteristics may have played a role in the result. Furthermore, even though the participant had suffered from flight phobia for many years without improvement, repeated exposure to the same visual stimuli and recall of affective memories could have desensitized her. This limitation was partly overcome by the regression analysis which established predictive relationships between brain activity and psychological distress measures independent of test-retest effects. Although the effects of the EFT intervention were quantified using psychometric and neurophysiological methods, the study did not provide any information regarding the neuropsychological processes mediating the efficacy of EFT. It was designed primarily to provide functional brain imaging insights on the psychophysiological correlates of EFT interventions, and provide preliminary data to be used in robust experimental designs such as randomized controlled trials.

\section{References}

[1] Attal, Y., Bhattacharjee, M., Yelnik, J., Cottereau, B., LEFÈVRE, J., OKADA, Y., Bardinet, E., Chupin, M., And BAILLET, S. Modeling and detecting deep brain activity with meg \& eeg. In 2007 29th Annual International Conference of the IEEE Engineering in Medicine and Biology Society (2007), IEEE, pp. 4937-4940.

[2] Attal, Y., And Schwartz, D. Assessment of subcortical source localization using deep brain activity imaging model with minimum norm operators: a meg study. PLoS One 8, 3 (2013), e59856.

[3] Bach, D., Groesbeck, G., StapleTon, P., Sims, R., Blickheuser, K., And Church, D. Clinical eft (emotional freedom techniques) improves multiple physiological markers of health. Journal of evidence-based integrative medicine 24 (2019), $2515690 X 18823691$.

[4] Baillet, S., Mosher, J. C., And LеАну, R. M. Electromagnetic brain mapping. IEEE Signal processing magazine 18, 6 (2001), 14-30.

[5] Baker, A. H., And Siegel, M. Emotional freedom techniques (eft) reduces intense fears: A partial replication and extension of wells et al. Energy Psychology: The- 
ory, Research, and Treatment 2, 2 (2010), $13-30$.

[6] BAKKeR, G. A bigger swamp is still a swamp: Comments on feinstein (2014). Energy Psychology: Theory, Research, and Treatment 6, 1 (2014), 44-47.

[7] Banks, S. J., EdDY, K. T. Angstadt, M., NAthan, P. J., AND PHAN, K. L. Amygdala-frontal connectivity during emotion regulation. Social cognitive and affective neuroscience 2, 4 (2007), 303-312.

[8] Bates, D., Maechler, M. Bolker, B., WAlker, S., ET AL. lme4: Linear mixed-effects models using eigen and s4. $R$ package version 1, 7 (2014), $1-23$.

[9] Bendamini, Y., And Hochberg, Y. Controlling the false discovery rate: a practical and powerful approach to multiple testing. Journal of the Royal statistical society: series B (Methodological) 57, 1 (1995), 289-300.

[10] Boyd, J., Rae, D., Thompson, J., Burns, B., Bourdon, K., Locke, B., AND REgier, D. Phobia: prevalence and risk factors. Social psychiatry and psychiatric epidemiology 25, 6 (1990), 314-323.

[11] $\mathrm{CHURCH}$, D. Clinical eft as an evidencebased practice for the treatment of psychological and physiological conditions. Psychology 4, 8 (2013), 645-654.

[12] Church, D. The EFT manual (3rd ed.). Santa Rosa, CA: Energy Psychology Press., 2013.

[13] Church, D., Feinstein, D., PAlmer-Hoffman, J., Stein, P. K., AND TRAnguCH, A. Empirically supported psychological treatments: The challenge of evaluating clinical innovations. The Journal of nervous and mental disease 202, 10 (2014), 699-709.

[14] Church, D., Yount, G., AND Brooks, A. J. The effect of emotional freedom techniques on stress biochemistry: a randomized controlled trial. The Journal of nervous and mental disease 200, 10 (2012), 891-896.

[15] Church, D., Yount, G., Rachlin, K., Fox, L., AND NELms, J. Epigenetic effects of ptsd remediation in veterans using clinical emotional freedom techniques: a randomized controlled pilot study. American Journal of Health Promotion 32, 1 (2018), $112-122$

[16] Clond, M. Emotional freedom techniques for anxiety: a systematic review with metaanalysis. The Journal of nervous and mental disease 204, 5 (2016), 388-395.

[17] Coelho, C. M., Lipp, O. V., MariNovic, W., Wallis, G., And Riek, $\mathrm{S}$. Increased corticospinal excitability induced by unpleasant visual stimuli. Neuroscience letters 481, 3 (2010), 135-138.

[18] Craig, G. The EFT manual. Elite Books, 2011.

[19] David, O., Kilner, J. M., AND FRISTON, K. J. Mechanisms of evoked and induced responses in meg/eeg. Neuroimage 31, 4 (2006), 1580-1591.

[20] DeAn, R. D., AND Whitaker, K. M. Fear of flying: Impact on the us air travel industry. Journal of Travel Research 21, 1 (1982), 7-17.

[21] Devilly, G. J. Power therapies and possible threats to the science of psychology and psychiatry. Australian and New Zealand Journal of Psychiatry 39, 6 (2005), 437-445.

[22] Doenrmann, O., Ghosh, S. S., Polli, F. E., ReYnolds, G. O., Horn, F., Keshavan, A., TriAntafyllou, C., SAYgin, Z. M., Whitfield-GABRieli, S., HofMANN, S. G., ET AL. Predicting treatment response in social anxiety disorder from functional magnetic resonance imaging. JAMA psychiatry 70, 1 (2013), 87-97.

[23] Duval, E. R., Javanbakht, A., AND LIBERZON, I. Neural circuits in anxiety and stress disorders: a focused review. Therapeutics and clinical risk management 11 (2015), 115.

[24] Ekeberg, Ø., Semberg, I., And Ellertsen, B. B. The prevalence of flight anxiety in norway. Nordisk Psykiatrisk Tidsskrift 43, 5 (1989), 443-448.

[25] Feinstein, D. Energy psychology: A review of the preliminary evidence. Psychotherapy: Theory, Research, Practice, Training 45, 2 (2008), 199.

[26] Feinstein, D. Facts, paradigms, and anomalies in the acceptance of energy psychology: A rejoinder to mccaslinŠs (2009) 
and pignotti and thyerŠs (2009) comments on feinstein (2008a). Educational Publishing Foundation (2009).

[27] Feinstein, D., And Eden, D. Six pillars of energy medicine. Alternative Therapies in Health and Medicine (2007).

[28] Feinstein, D., And Eden, D. Six pillars of energy medicine: Clinical strengths of a complementary paradigm. alternative therapies, 14 (1), 44-54. Six Pillars of Energy Medicine Clinical Strengths of a Complementary Paradigm David Feinstein, Ph. D., and Donna Eden ABSTRACT (2008).

[29] Fontaine, R., Breton, G., Déry, R., Fontaine, S., And Elie, R. Temporal lobe abnormalities in panic disorder: an mri study. Biological Psychiatry 27, 3 (1990), 304-310.

[30] Gallo, F. P. Energy psychology. CRC Press, 2004.

[31] Gaudiano, B. A., Brown, L. A., AND Miller, I. W. Tapping their patientsŠ problems away? characteristics of psychotherapists using energy meridian techniques. Research on Social Work Practice 22, 6 (2012), 647-655.

[32] Goldin, P. R., McRae, K., Ramel, W., And Gross, J. J. The neural bases of emotion regulation: reappraisal and suppression of negative emotion. Biological psychiatry 63, 6 (2008), 577-586.

[33] Gray, J. A. Brain systems that mediate both emotion and cognition. Cognition \& emotion 4, 3 (1990), 269-288.

[34] Grèzes, J., Tucker, M., Armony, J., Ellis, R., AND PAssingham, R. E. Objects automatically potentiate action: an fmri study of implicit processing. European Journal of Neuroscience 17, 12 (2003), 2735-2740.

[35] Grezes, J., Valabregue, R., Gholipour, B., And Chevallier, C. A direct amygdala-motor pathway for emotional displays to influence action: A diffusion tensor imaging study. Human brain mapping 35, 12 (2014), 5974-5983.

[36] Gurret, J.-M., Caufour, C., PALMER-HOFFMAN, J., AND CHuRCH, D. Post-earthquake rehabilitation of clinical ptsd in haitian seminarians. Energy Psychology: Theory, Research, and Treatment 4, 2 (2012), 26-34.
[37] Hajcak, G., Molnar, C., George, M. S., Bolger, K., Koola, J., And NAHAS, Z. Emotion facilitates action: a transcranial magnetic stimulation study of motor cortex excitability during picture viewing. Psychophysiology 44, 1 (2007), 91-97.

[38] HeimberG, R. G., Liebowitz, M. R., Hope, D. A., Schneier, F. R., Holt, C. S., Welkowitz, L. A., Juster, H. R., Campeas, R., Bruch, M. A., Cloitre, M., ET AL. Cognitive behavioral group therapy vs phenelzine therapy for social phobia: 12-week outcome. Archives of General Psychiatry 55, 12 (1998), 1133-1141.

[39] Hermann, A., Schäfer, A., WalTER, B., StARK, R., VAitl, D., And Schienle, A. Diminished medial prefrontal cortex activity in blood-injectioninjury phobia. Biological psychology 75, 2 (2007), 124-130.

[40] Hermann, A., Schäfer, A., WalTer, B., Stark, R., Vaitl, D., And SCHIEnle, A. Emotion regulation in spider phobia: role of the medial prefrontal cortex. Social cognitive and affective neuroscience 4, 3 (2009), 257-267.

[41] Holmes, E. A., And Mathews, A. Mental imagery and emotion: A special relationship? Emotion 5, 4 (2005), 489.

[42] Ipser, J. C., Singh, L., And Stein, D. J. Meta-analysis of functional brain imaging in specific phobia. Psychiatry and clinical neurosciences 67, 5 (2013), 311-322.

[43] Karatzias, T., Power, K., Brown, K., MCGoldrick, T., BEGuM, M., Young, J., Loughran, P., Chouliara, Z., And Adams, S. A controlled comparison of the effectiveness and efficiency of two psychological therapies for posttraumatic stress disorder: eye movement desensitization and reprocessing vs. emotional freedom techniques. The Journal of nervous and mental disease 199, 6 (2011), 372-378.

[44] Lang, P., And Bradley, M. M. The international affective picture system (iaps) in the study of emotion and attention. Handbook of emotion elicitation and assessment 29 (2007).

[45] Levin, J. Energy healers: who they are and what they do. Explore 7, 1 (2011), 13-26. 
[46] Levinson, H. N. A cerebellar-vestibular explanation for fears/phobias: hypothesis and study. Perceptual and Motor Skills 68, 1 (1989), 67-84.

[47] Mahara J, M. Differential gene expression after emotional freedom techniques (eft) treatment: A novel pilot protocol for salivary mrna assessment. Energy Psychol Theory Res Treat 8, 1 (2016), 17-32.

[48] Malejko, K., Abler, B., Plener, P. L., AND StRAUB, J. Neural correlates of psychotherapeutic treatment of posttraumatic stress disorder: A systematic literature review. Frontiers in psychiatry 8 (2017), 85.

[49] McCaslin, D. L. A review of efficacy claims in energy psychology. Psychotherapy: Theory, Research, Practice, Training (2009).

[50] Morawetz, C., Bode, S., BAUDEWIG, J., AND HEEKEREN, H. R. Effective amygdala-prefrontal connectivity predicts individual differences in successful emotion regulation. Social cognitive and affective neuroscience 12, 4 (2017), 569-585.

[51] Nelms, J. A., And Castel, L. A systematic review and meta-analysis of randomized and nonrandomized trials of clinical emotional freedom techniques (eft) for the treatment of depression. Explore 12, 6 (2016), 416-426.

[52] Neuper, C., Wörtz, M., AND Pfurticheller， G. Erd/ers patterns reflecting sensorimotor activation and deactivation. Progress in brain research 159 (2006), 211-222.

[53] ÖHMAN, A. The role of the amygdala in human fear: automatic detection of threat. Psychoneuroendocrinology 30, 10 (2005), 953958.

[54] Oliveri, M., Babiloni, C., Filippi, M., Caltagirone, C., Babiloni, F., Cicinelli, P., Traversa, R., Palmieri, M., AND Rosini, P. Influence of the supplementary motor area on primary motor cortex excitability during movements triggered by neutral or emotionally unpleasant visual cues. Experimental brain research 149, 2 (2003), 214-221.

[55] Paquette, V., Lévesque, J., MenSOUR, B., LEROUX, J.-M., BEAUDoIn, G., Bourgouin, P., AND
BEA UREGARD, M. âĂIJchange the mind and you change the brainâĂİ: effects of cognitive-behavioral therapy on the neural correlates of spider phobia. Neuroimage 18, 2 (2003), 401-409.

[56] Peres, J. F., Foerster, B., SanTANA, L. G., FErEirA, M. D., Nasello, A. G., SAvoia, M., Moreira-Almeida, A., AND LedERMAN, H. Police officers under attack: resilience implications of an fmri study. Journal of psychiatric research 45, 6 (2011), 727734 .

[57] Peres, J. F., Newberg, A. B., Mercante, J. P., Simao, M., AlBuquerque, V. E., Peres, M. J., And Nasello, A. G. Cerebral blood flow changes during retrieval of traumatic memories before and after psychotherapy: a spect study. Psychological medicine 3\%, 10 (2007), 1481-1491.

[58] Pfurtacheller， G. Spatiotemporal erd/ers patterns during voluntary movement and motor imagery. In Supplements to Clinical neurophysiology, vol. 53. Elsevier, 2000, pp. 196-198.

[59] Pfurtscheller, G. Functional brain imaging based on erd/ers. Vision research 41, 10-11 (2001), 1257-1260.

[60] Phan, K. L., Coccaro, E. F., Angstadt, M., Kreger, K. J., MAyberG, H. S., Liberzon, I., AND Stein, M. B. Corticolimbic brain reactivity to social signals of threat before and after sertraline treatment in generalized social phobia. Biological Psychiatry 73, 4 (2013), 329-336.

[61] Pichon, S., DE Gelder, B., AND Grezes, J. Threat prompts defensive brain responses independently of attentional control. Cerebral Cortex 22, 2 (2011), 274285.

[62] Pignotti, M., And Thyer, B. Some comments on" energy psychology: A review of the evidence": Premature conclusions based on incomplete evidence? Psychotherapy: Theory, Research, Practice, Training (2009).

[63] Rancour, P. The emotional freedom technique: Finally, a unifying theory for the practice of holistic nursing, or too good to be true? Journal of Holistic Nursing 35, 4 (2017), 382-388. 
[64] Rive, M. M., van Rooijen, G., Veltman, D. J., Phillips, M. L., SChene, A. H., AND Ruhe, H. G. Neural correlates of dysfunctional emotion regulation in major depressive disorder. a systematic review of neuroimaging studies. Neuroscience 85 Biobehavioral Reviews 37, 10 (2013), 2529-2553.

[65] Sacchetti, B., Baldi, E., LorenZini, C. A., AND Bucherelli, C. Cerebellar role in fear-conditioning consolidation. Proceedings of the National Academy of Sciences 99, 12 (2002), 8406-8411.

[66] Sacchetti, B., Scelfo, B., AND Strata, P. The cerebellum: synaptic changes and fear conditioning. The Neuroscientist 11, 3 (2005), 217-227.

[67] Salas, M. M., Brooks, A. J., AND Rowe, J. E. The immediate effect of a brief energy psychology intervention (emotional freedom techniques) on specific phobias: A pilot study. Explore: The Journal of Science and Healing 7, 3 (2011), 155-161.

[68] Schweckendiek, J., Klucken, T., Merz, C. J., TABbert, K., WALTer, B., Aмвасн, W., VAitl, D., AND STARK, R. Weaving the (neuronal) web: fear learning in spider phobia. Neuroimage 54, 1 (2011), 681-688.

[69] Sebastian, B., And Nelms, J. The effectiveness of emotional freedom techniques in the treatment of posttraumatic stress disorder: A meta-analysis. Explore 13, 1 (2017), 16-25.

[70] Smith, E., And Kosslyn, S. Cognitive psychology: Mind and brain. 2007, 2007.

[71] Somers, J. M., Goldner, E. M., Waraich, P., AND Hsu, L. Prevalence and incidence studies of anxiety disorders: a systematic review of the literature. The Canadian Journal of Psychiatry 51, 2 (2006), 100-113.

[72] Strata, P., Scelfo, B., And SaCCHETTI, B. Involvement of cerebellum in emotional behavior. Physiol Res 60, Suppl 1 (2011), S39-S48.

[73] Strick, P. L., Dum, R. P., AND FIEZ, J. A. Cerebellum and nonmotor function. Annual review of neuroscience 32 (2009), 413-434.

[74] Tadel, F., Baillet, S., Mosher, J. C., Pantazis, D., And Leahy,
R. M. Brainstorm: a user-friendly application for meg/eeg analysis. Computational intelligence and neuroscience 2011 (2011), 8.

[75] Taylor, C. T., Aupperle, R. L., Flagan, T., Simmons, A. N., Amir, N., Stein, M. B., AND PAulus, M. P. Neural correlates of a computerized attention modification program in anxious subjects. Social cognitive and affective neuroscience 9, 9 (2013), 1379-1387.

[76] Tém, R. C., eт AL. R: A language and environment for statistical computing.

[77] Tucker, M., And Ellis, R. Action priming by briefly presented objects. Acta psychologica 116, 2 (2004), 185-203.

[78] VAn GerWen, L. J., SPINHoven, P., Diekstra, R. F., AND VAn DyCK, R. People who seek help for fear of flying: Typology of flying phobics. Behavior Therapy 28, 2 (1997), 237-251.

[79] Van Gerwen, L. J., Spinhoven, P., Van Dyck, R., And Diekstra, R. F. Construction and psychometric characteristics of two self-report questionnaires for the assessment of fear of flying. Psychological assessment 11, 2 (1999), 146.

[80] Wager, T. D., Davidion, M. L., Hughes, B. L., Lindquist, M. A., AND OChsner, K. N. Prefrontalsubcortical pathways mediating successful emotion regulation. Neuron 59, 6 (2008), 1037-1050.

[81] Wells, S., Polglase, K., ANDrews, H. B., CARrington, P., AND BAKER, A. H. Evaluation of a meridian-based intervention, emotional freedom techniques (eft), for reducing specific phobias of small animals. Journal of Clinical Psychology 59, 9 (2003), 943-966.

[82] Wilhelm, F. H., And Roth, W. T. Clinical characteristics of flight phobia. Journal of anxiety disorders 11, 3 (1997), 241-261.

[83] Wolpe. The practice of behavior therapy. Pergamon, 1973.

[84] X U, R. Measuring explained variation in linear mixed effects models. Statistics in medicine 22, 22 (2003), 3527-3541. 